Supplement of The Cryosphere, 13, 1547-1564, 2019

https://doi.org/10.5194/tc-13-1547-2019-supplement

(c) Author(s) 2019. This work is distributed under

the Creative Commons Attribution 4.0 License.

(c) (1)

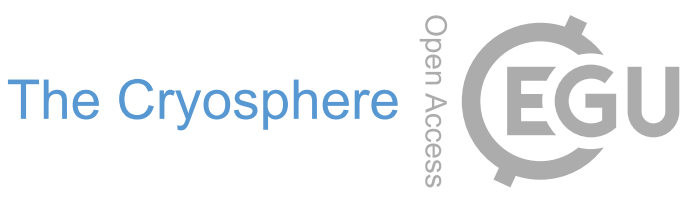

Supplement of

\title{
Regional grid refinement in an Earth system model: impacts on the simu- lated Greenland surface mass balance
}

Leonardus van Kampenhout et al.

Correspondence to: Leonardus van Kampenhout (l.vankampenhout@uu.nl)

The copyright of individual parts of the supplement might differ from the CC BY 4.0 License. 
Supplementary material

\section{Contents of this file}

- Figure S1

- Figure S2

- Figure S3

- Figure S4

- Figure S5

- Figure S6

- Figure S7

- Figure S8

- Figure S9

- Figure S10

- Figure S11
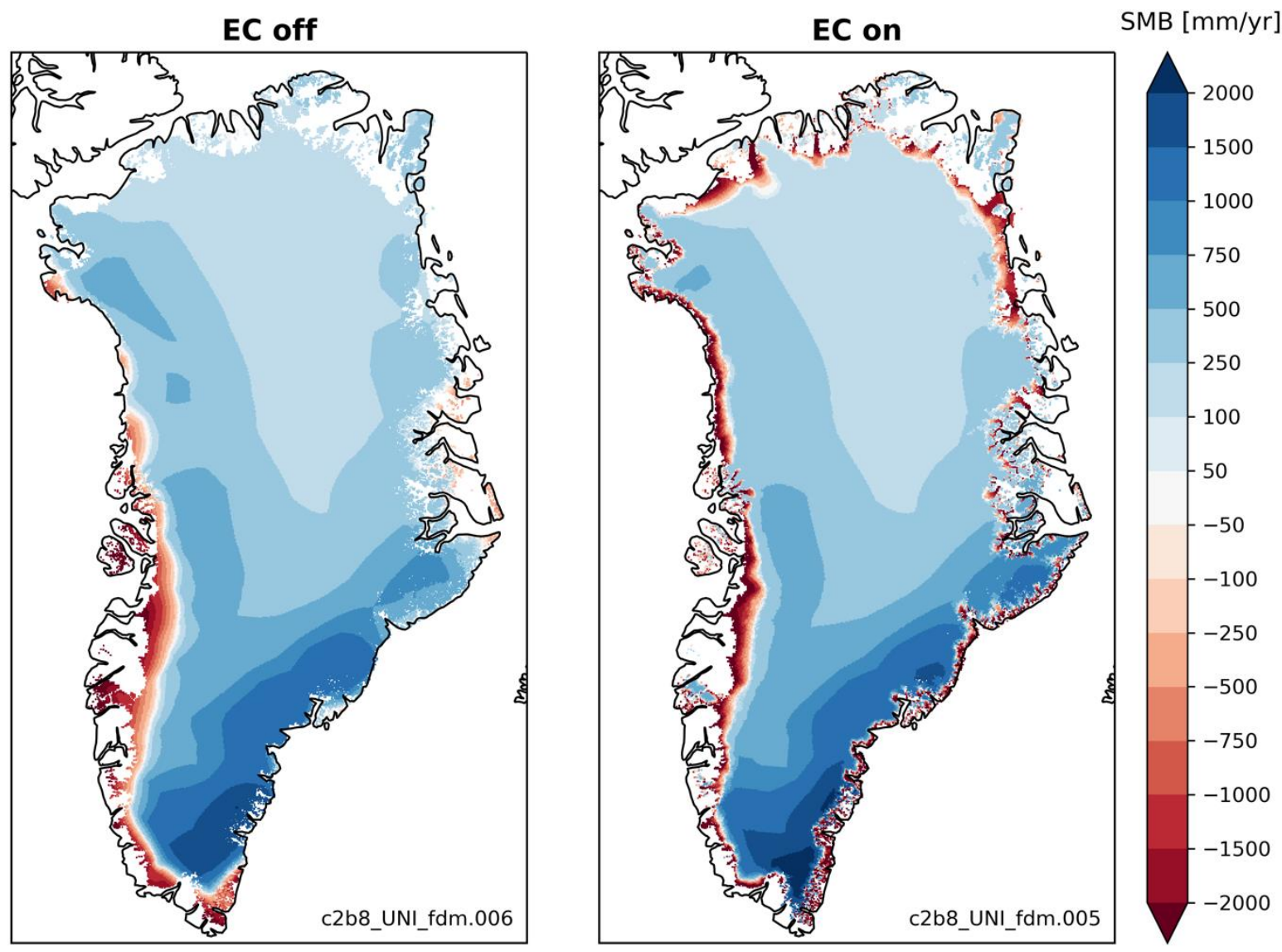
Figure S1. Annual mean SMB at $4 \mathrm{~km}$ over the period 1980-1999 in two 1-degree CESM simulations. Left panel: a simulation identical in setup to Uniform CESM, except that both EC lapse rates (Section 2.3) are set to zero. Right panel: simulation Uniform CESM.

VR-CESM55 - Uniform

$10 \mathrm{~m}$ wind speed

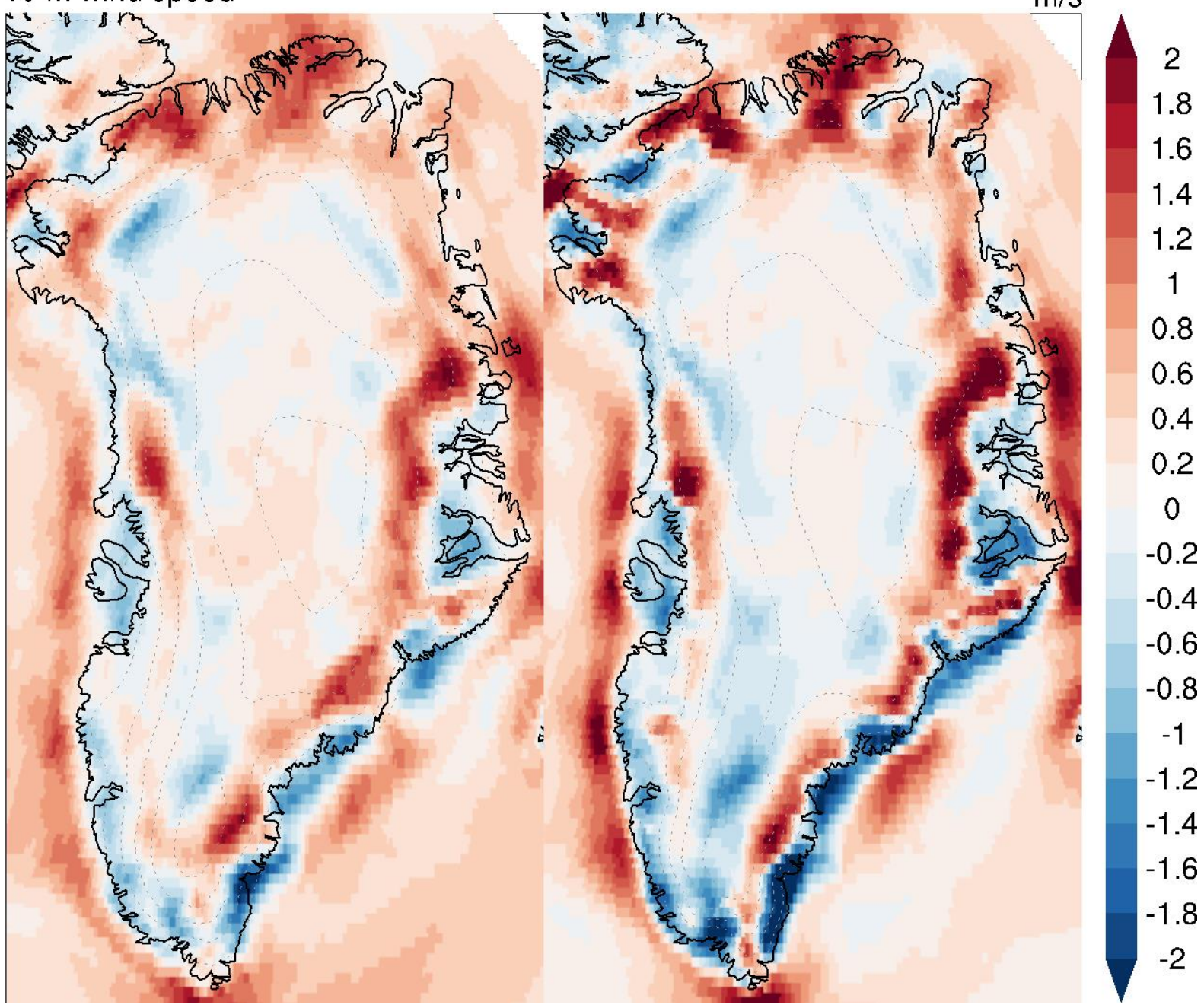

Figure S2: Summer (JJA) anomalies of CESM (CAM-SE) 10-m wind speed over the period 1980-1999. Prior to subtraction, all data have been regridded to a common regular mesh of $0.25^{\circ}$ using bi-linear interpolation. 


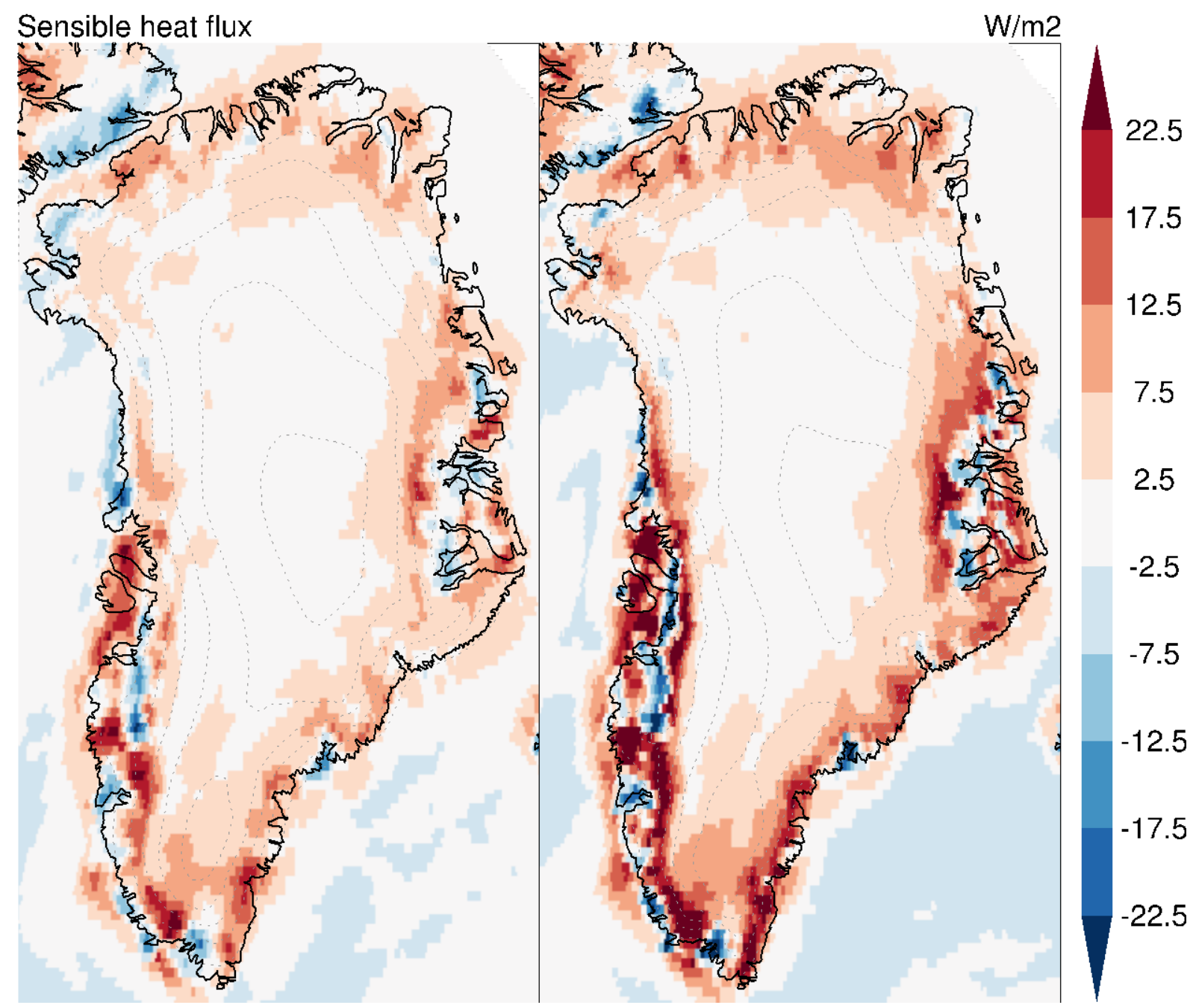

Figure S3: Summer (JJA) anomalies of CESM (CAM-SE) sensible heat flux (+ towards the surface) over the period 1980-1999. Prior to subtraction, all data have been regridded to a common regular mesh of $0.25^{\circ}$ using bi-linear interpolation. Higher values in VR-CESM are probably due to the higher surface wind speeds that enhance turbulence (Figure S2). 
VR-CESM55 - Uniform

VR-CESM28 - Uniform

Downwelling shortwave radiation

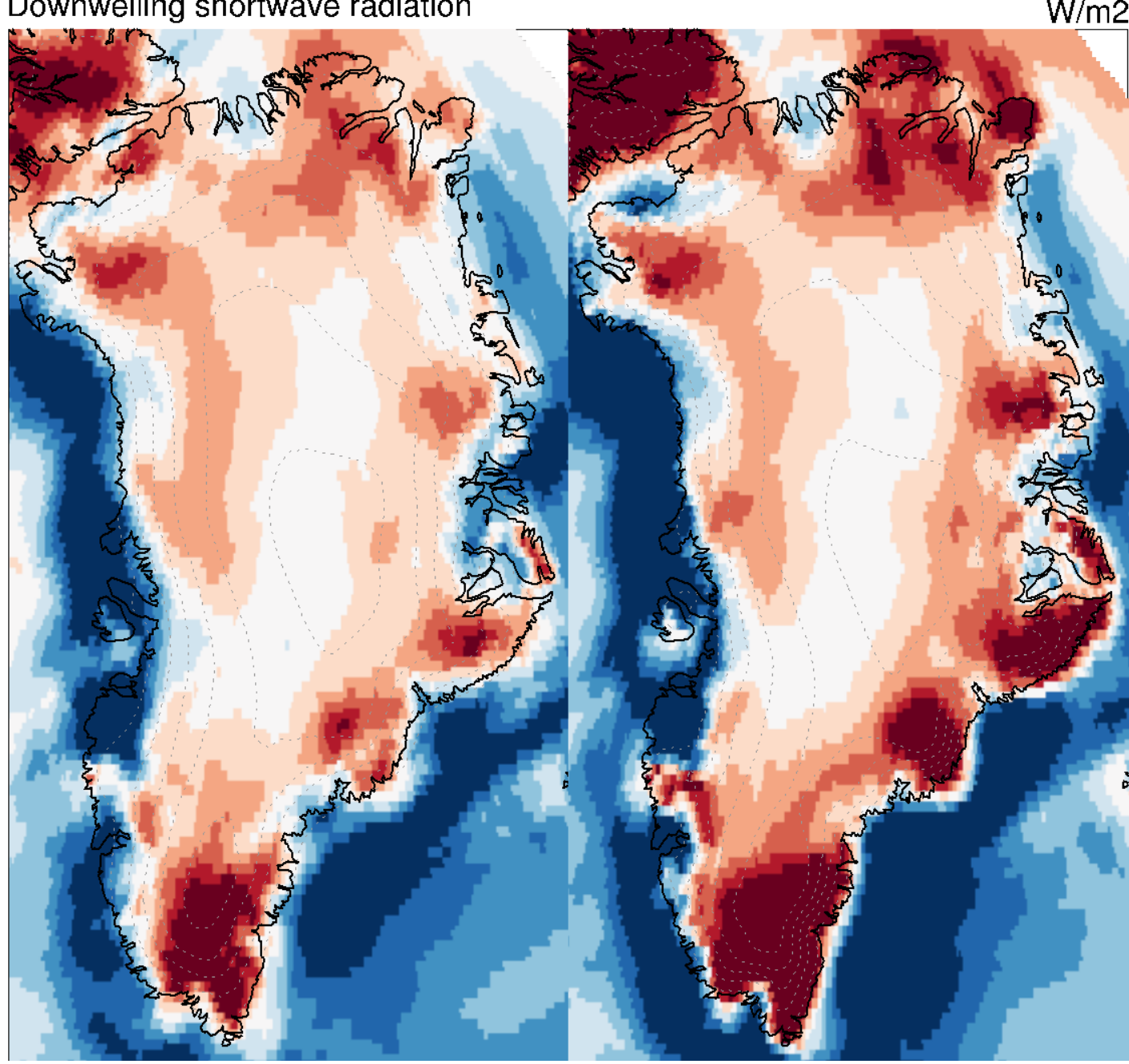

22.5

17.5

12.5

7.5

2.5

$-2.5$

$-7.5$

$-12.5$

$-17.5$

$-22.5$

Figure S4: Summer (JJA) anomalies of CESM (CAM-SE) downwelling surface shortwave radiation over the period 1980-1999. Prior to subtraction, all data have been regridded to a common regular mesh of $0.25^{\circ}$ using bi-linear interpolation. 

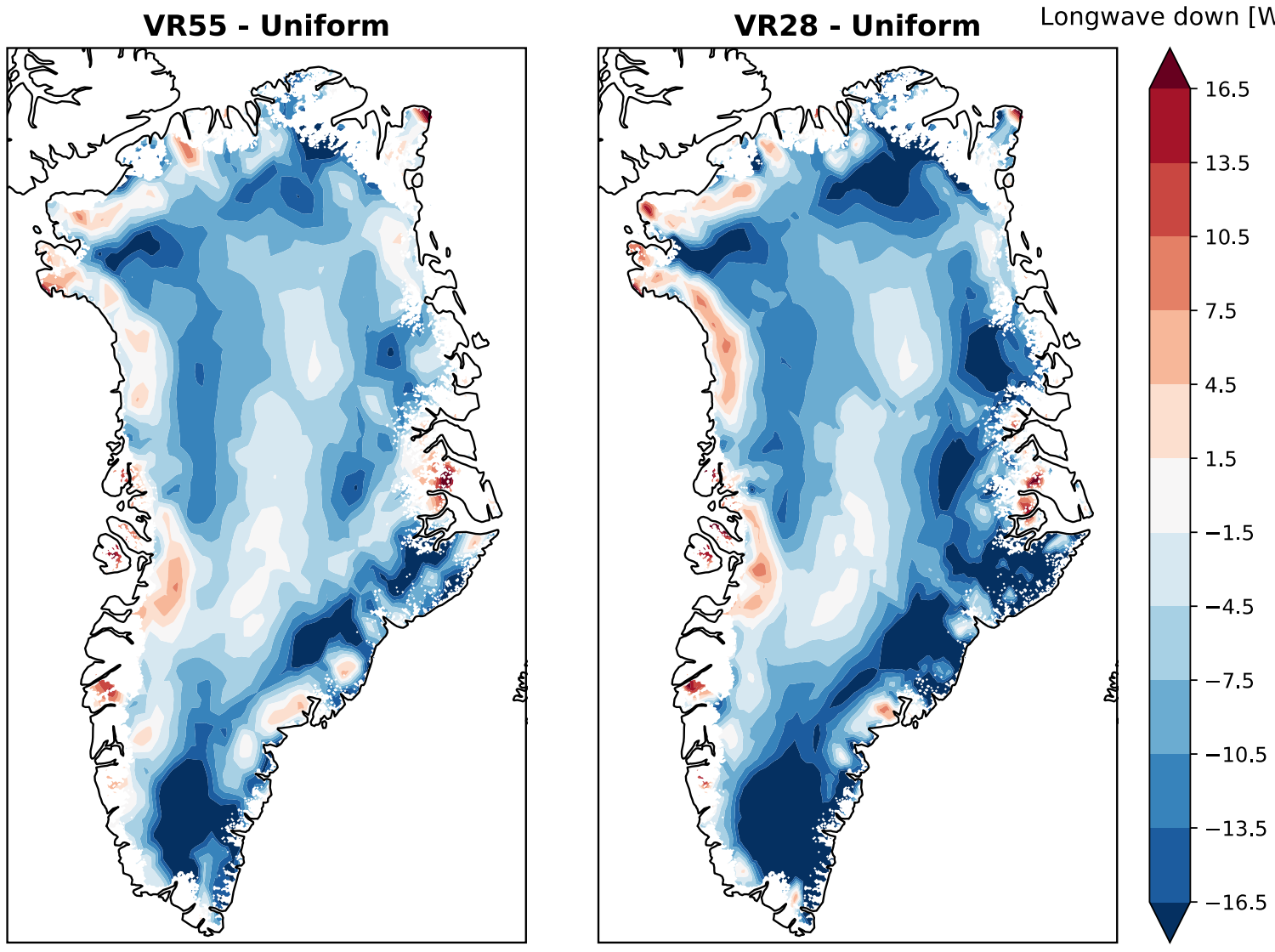

Figure S5. Summer (JJA) anomalies of CESM (CLM) downwelling longwave radiation (after downscaling) over glaciated landunits, relative to the coarse resolution reference simulation (Uniform CESM) over the period 1980-1999. Data have been downscaled to $4 \mathrm{~km}$ using EC output. 


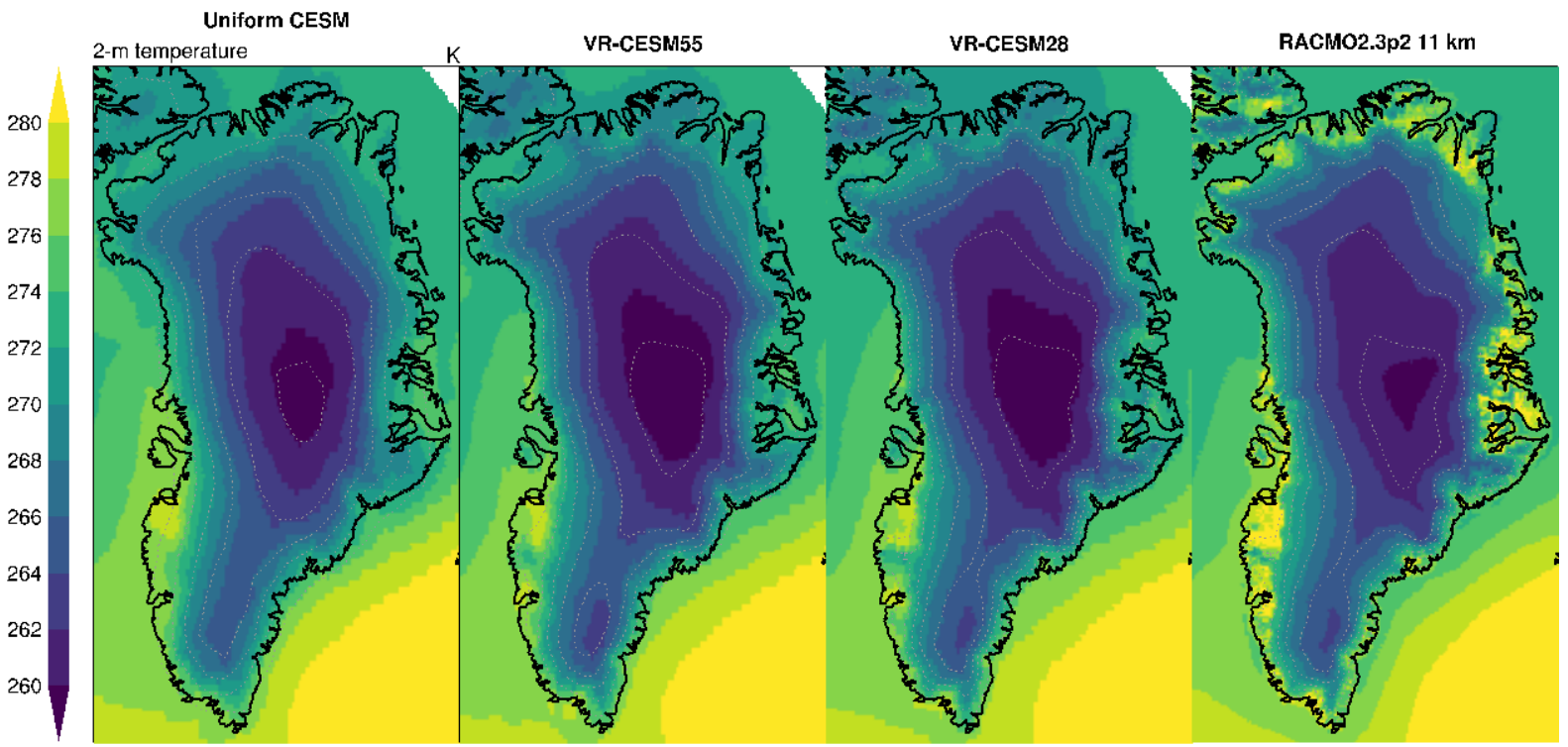

Figure S6. Summer (JJA) 2-m air temperature in CESM (CAM-SE) and RACMO2. All data have been regridded to a common regular mesh of $0.25^{\circ}$ using bi-linear interpolation.

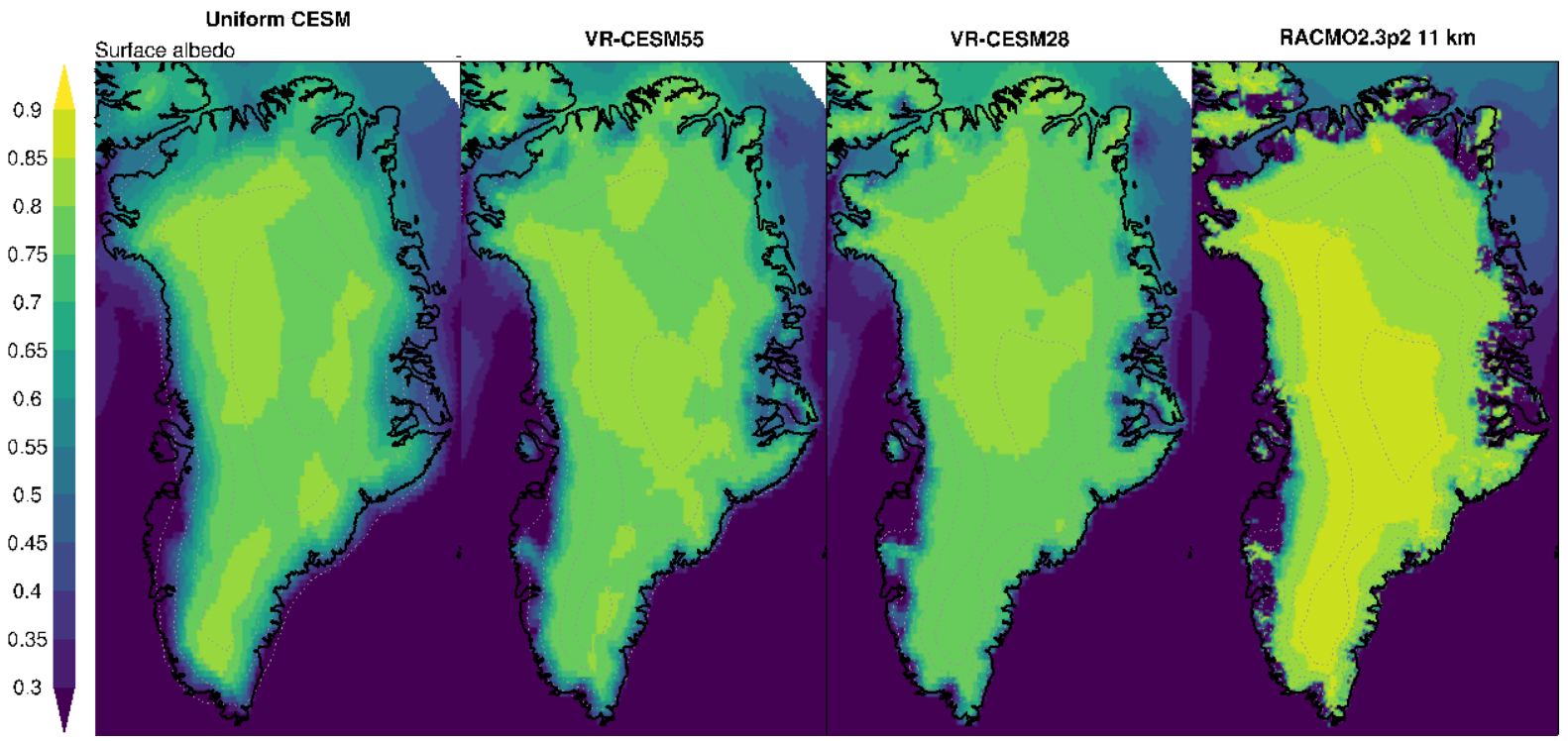

Figure S7. Summer (JJA) surface albedo in CESM (CAM-SE) and RACMO2. All data have been regridded to a common regular mesh of $0.25^{\circ}$ using bi-linear interpolation. 


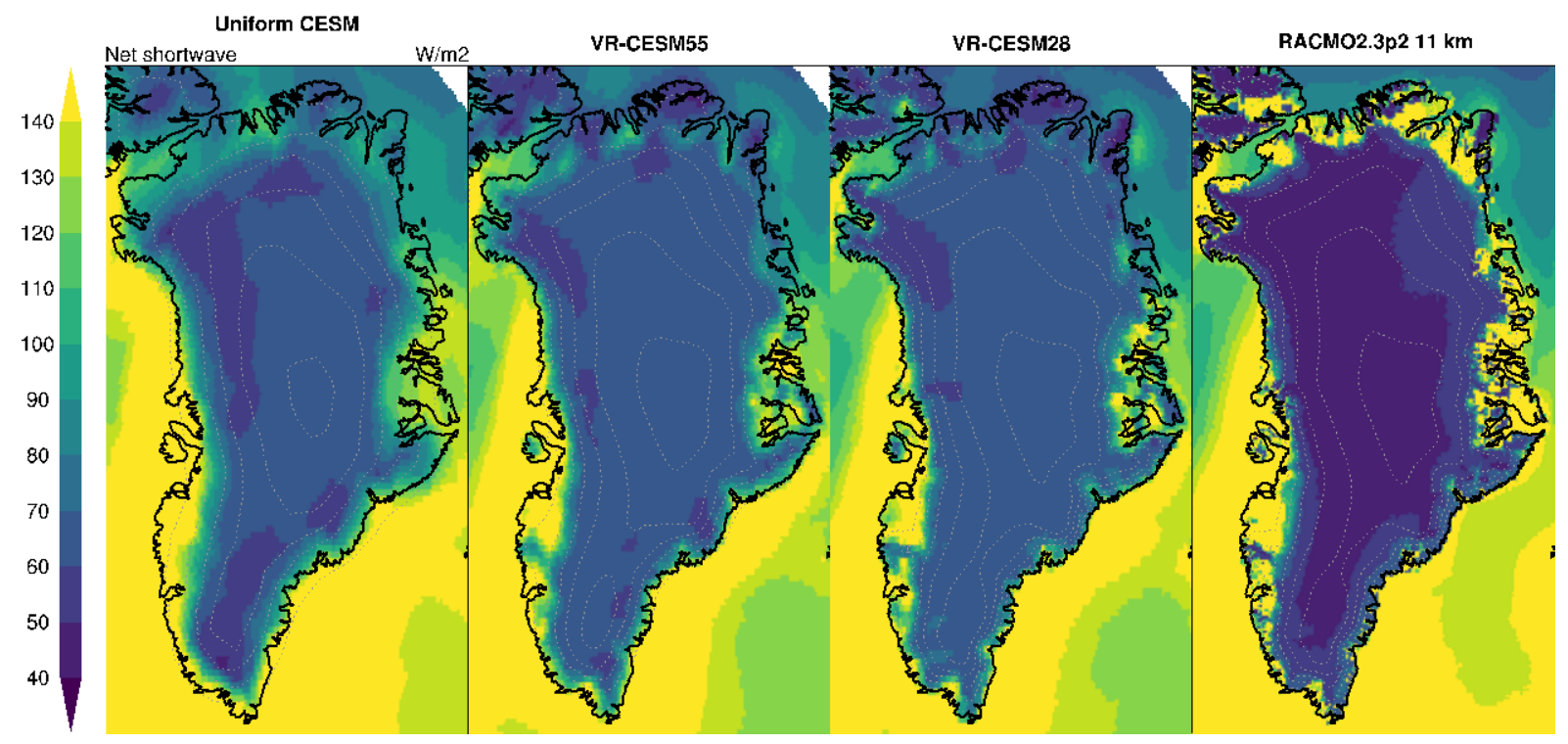

Figure S8. Summer (JJA) net shortwave surface radiation in CESM (CAM-SE) and RACMO2. All data have been regridded to a common regular mesh of $0.25^{\circ}$ using bi-linear interpolation.

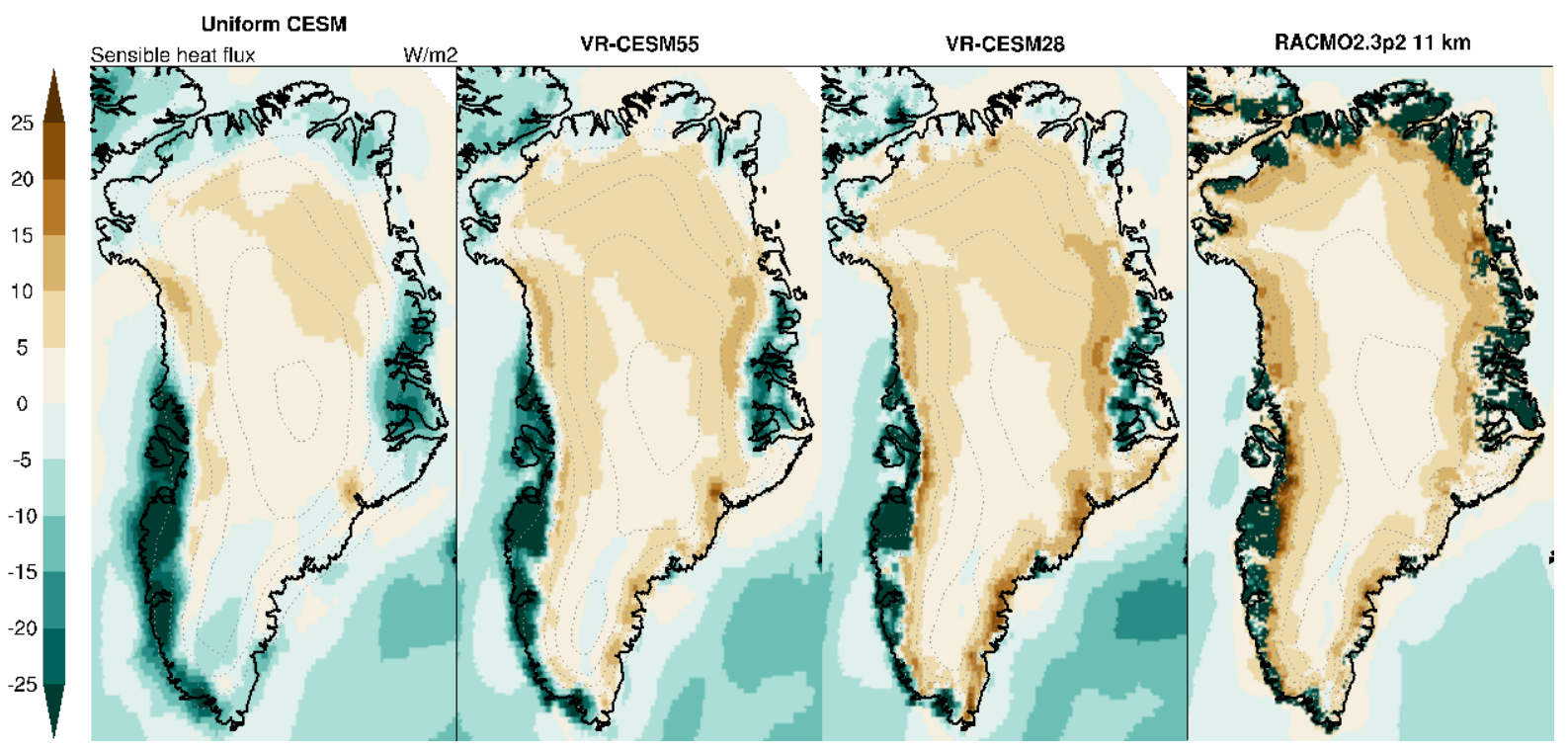

Figure S9. Summer (JJA) turbulent sensible heat flux in CESM (CAM-SE) and RACMO2. All data have been regridded to a common regular mesh of $0.25^{\circ}$ using bi-linear interpolation. 


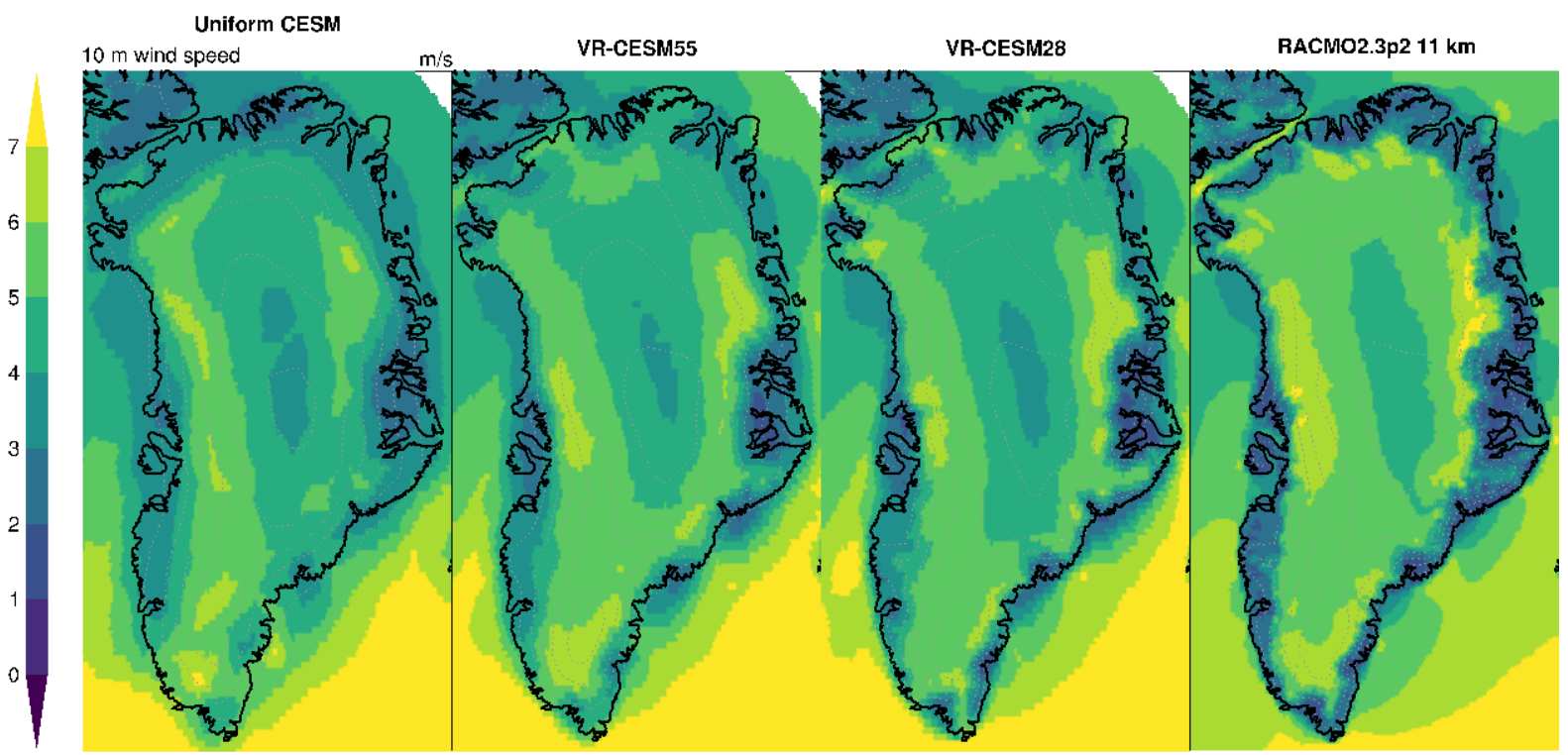

Figure S10. Summer (JJA) 10-m wind speed in CESM (CAM-SE) and RACMO2. All data have been regridded to a common regular mesh of $0.25^{\circ}$ using bi-linear interpolation.

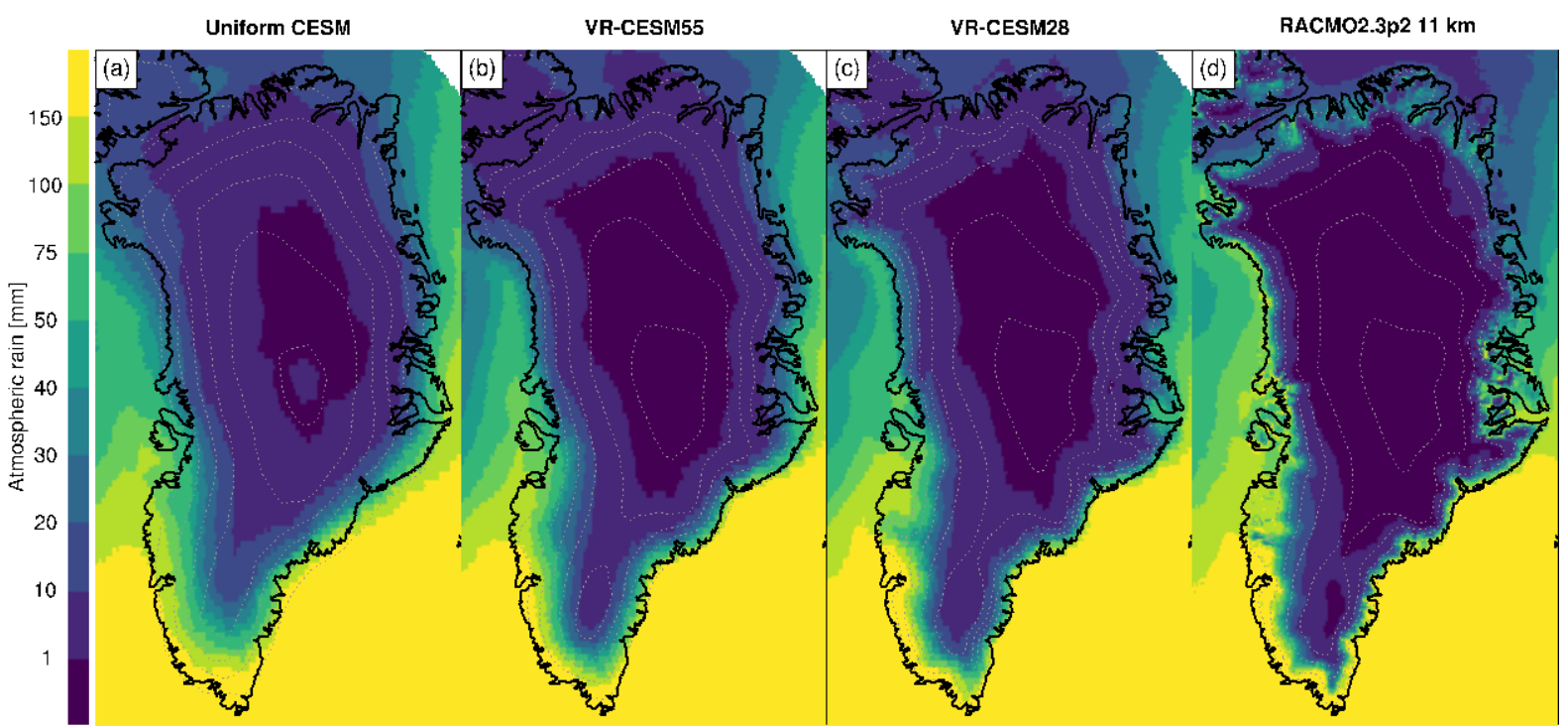

Figure S11. Summer (JJA) rainfall in CESM (CAM-SE) and RACMO2. All data have been regridded to a common regular mesh of $0.25^{\circ}$ using bi-linear interpolation. 